Meta

Journal des traducteurs

Translators' Journal

\title{
Le mémoire de terminologie
}

\section{Roger Goffin}

Volume 23, numéro 4, décembre 1978

URI : https://id.erudit.org/iderudit/002932ar

DOI : https://doi.org/10.7202/002932ar

Aller au sommaire du numéro

Éditeur(s)

Les Presses de l'Université de Montréal

ISSN

0026-0452 (imprimé)

1492-1421 (numérique)

Découvrir la revue

Citer cet article

Goffin, R. (1978). Le mémoire de terminologie. Meta, 23(4), 303-307.

https://doi.org/10.7202/002932ar

Ce document est protégé par la loi sur le droit d'auteur. L'utilisation des services d'Érudit (y compris la reproduction) est assujettie à sa politique d'utilisation que vous pouvez consulter en ligne.

https://apropos.erudit.org/fr/usagers/politique-dutilisation/ 


\section{DROBLEMES ET SOLUTIONS}

\section{LE MÉMOIRE DE TERMINOLOGIE}

\section{UNE FORME DE RAPPROCHEMENT ENTRE THÉORIE ET PRATIQUE DE LA TERMINOLOGIE}

\section{Recherches en traduction et en terminologie}

De même que le titre universitaire de «licencié en philosophie et lettres » est, en Belgique, subordonné à la rédaction d'un travail personnel appelé mémoire de licence, le grade de «licencié-traducteur» ne peut être obtenu, au terme d'un cycle de quatre années d'études, qu'après présentation d'un travail de recherche rédigé dans l'une des deux langues modernes choisies. Il doit se rapporter à un sujet d'ordre linguistique, économique, social ou culturel. Les mêmes exigences existent dans d'autres pays, assorties il est vrai de modalités différentes.

Traditionnellement, l'université est un lieu de recherche intellectuelle et les enseignements qu'elle dispense ont toujours pour but d'encourager les jeunes esprits à s'intégrer à la recherche sous ses formes les plus diverses. Les instituts de traduction sont eux aussi appelés à assumer la double fonction d'enseignement et de recherche malgré leur finalité professionnelle.

Les recherches en traduction peuvent aller vers la recherche fondamentale de nature abstraite et théorique. Leur but est alors d'aider à construire un corps de doctrine cohérent permettant d'interpréter, au moyen de lois plus générales; les phénomènes mis en jeu dans l'activité traduisante. Il se développe un type de recherche qui reste en contact avec ce que certains ont appelé les «questions fondationnelles ». La discipline repose elle ausṣi sur un certain nombre d'axiomes d'ordre épistémologique et méthodologique.

Un vaste champ d'investigation s'est ouvert aux jeunes chercheurs en traduction: 1) l'analyse de la traduction-action (acte et processus), 2) l'analyse des textes soumis à la traduction, 3) l'analyse de la traduction-produit, 4) la confrontation des systèmes linguistiques, 5 ) l'analyse d'une langue de spécialité.

\section{Le mémoire de terminologie}

Toutefois, les théories ne peuvent s'élaborer qu'à partir de données patiemment accumulées par les observateurs et collecteurs. C'est là que ce dessine une forme de recherche, à la fois plus modeste et plus directement orientée vers des buts pratiques. Elle se concrétise par la confection d'un mémoire de terminologie. Ce type de travail s'insère aussi 
dans l'esprit et le contexte des enseignements dispensés dans les départements de langues et de linguistique.

L'objet du mémoire de terminologie, de caractère pragmatique, est d'étudier de manière contrastive une langue de spécialité ou un sous-secteur d'une langue de spécialité à partir d'un corpus recueilli en deux ou plusieurs langues. Le travail comporte deux grandes phases : 1) la phase de préparation ( choix du sujet ), 2) la phase d'exécution, qui comprend la collecte des matériaux, le recensement du vocabulaire, l'établissement des équivalences (choix des termes, appariement), la présentation du corpus et des index.

Le travail peut être enrichi par une étude critique des sources et comprendre une brève étude sur les aspects linguistiques du vocabulaire (technolecte) étudié.

\section{La phase de préparation du mémoire de terminologie: le choix du sujet}

Comme l'étudiant ne dispose en général que d'un temps limité pour mener à bien le travail, il importe que le sujet soit spécifique, restreint et bien délimité. Bien souvent, le thème est choisi parmi les suggestions émises par l'étudiant en fonction de ses affinités électives (vocabulaire de l'aéromodélisme ou de la stéréophonie) ou en fonction de sa possibilité de se procurer de la documentation dans un secteur spécifique. Avant d'établir un choix définitif, les professeurs pourraient utilement consulter la liste des sujets de recherche proposés par les Services de terminologie des Institutions nationales ou internationales. Les Services de terminologie et les Établissements d'enseignement spécialisé sont d'ailleurs arrivés à un point où le risque existe que chacun sécrète ses propres produits sans trop se soucier des autres et travaille un peu en franc-tireur.

La délimitation du sujet se fera au fur et à mesure de la progression du travail. L'étudiant qui a choisi de traiter la terminologie de l'énergie solaire se verra contraint, à mesure qu'il compulse ses dossiers documentaires, de ne retenir par exemple que les collecteurs solaires et leur application dans l'habitat, d'autant que souvent le mémoire sinscrit dans un projet de travail collectif plus vaste et plus suivi.

\section{La phase d'exécution}

\subsection{La collecte des matériaux terminologiques et documentaires}

Le sujet une fois fixé, le travail commence par la collecte des matériaux documentaires. En général, toute publication spécialisée originale se rapportant au sujet doit être retenue pour la compilation. Vu le grand nombre de publications existant, l'étudiant se trouvera submergé par le flot d'informations. Les publications bibliographiques elles-mêmes ont pris des proportions démesurées. L'étudiant s'initiera en tout cas au domaine spécialisé, fera des incursions dans le domaine du spécialiste par des lectures attentives. Il se fera aider par son promoteur et tout spécialiste compétent. Il serait utile qu'il rédige un texte liminaire suivi qui décrive la technique étudiée et en explique le fonctionnement. Les sources écrites seront, si possible, complétées par des entrevues et des visites qui renforceront les contacts avec les gens du métier. Dès ce stade d'élaboration, l'étudiant prendra conscience des différents niveaux de la langue de spécialité : langue savante, jargon de laboratoire, terme normalisé. Le recensement ne commence que dans la seconde phase. 


\subsection{Le recensement}

C'est la phase essentielle et deux questions se posent:

a) que faut-il recenser?

b) comment faut-il recenser?

Le relevé du vocabulaire implique nécessairement un choix dans le flot de termes qui se présentent au lecteur. Au début, il sera malaisé de déterminer les critères de ce choix et il conviendrait de travailler suivant une méthode maximaliste. Généralement, le vocabulaire se décante spontanément. Le vocabulaire spécifique est sélectionné et relevé avec les distinctions suivantes: 1 ) langue technique, normalisée ou non, 2) jargon du technicien à pied d'œuvre, 3 ) termes d'usage courant qui, dans la langue du métier, peuvent avoir une signification particulière. Il se produit une sorte d'interaction entre le vocabulaire usuel et le vocabulaire de spécialité. L'étudiant procèdera de manière phénoménologique, enregistrant l'usage fixé par les textes et donnant un miroir de ce qui existe en dehors de tout souci de normalisation. La démarche restera purement sémasiologique et prendra appui sur des définitions. Le glossaire idéal devrait être débarrassé des mots techniques courants, donner à chaque fois une définition succincte, tenir compte des différences existant entre pays différents de même langue, donner le plus possible de termes rares et réellement spécifiques.

\subsection{L'établissement des équivalences}

Le dépouillement terminologique comparé comprend le repérage et la mise en parallèle de termes équivalents tirés de la littérature spécialisée originale. Cette confrontation des «performances " linguistiques n'est pas aisée d'autant que, parmi les équivalents potentiels, il y a lieu de fournir l'équivalent optimal, la correspondance absolue étant le plus souvent impossible. On constate, en effet, que les découpages de la réalité extralinguistique pratiqués par les langues de spécialité ne sont que rarement superposables et que l'on retrouve dans les terminologies les mêmes accidents que ceux qui frappent les mots du vocabulaire commun, notamment incohérence et polysémie. Un technolecte ne se réduit pas à un inventaire de mots dont il suffirait de changer l'étiquette pour passer d'une langue à l'autre.

Il serait sans doute fastidieux de faire un relevé complet des modèles d'appariement. Nous retiendrons ici sept modèles.

Cas no 1

Le premier est prétendument caractéristique pour le vocabulaire technique: un terme monosémique ( terminologisme) de la LD correspond à un terme monosémique de la LA. Une correspondance bi-univoque existe et le passage peut s'effectuer sans recours au dénoté.

\section{Cas no 2}

Un classème de la LD, au contenu sémantique démantelé, correspond dans la LA à des termes spécifiques et individualisables (ex.: aménagement, patrimoine, recyclage).

Cas no 3

Un terme désignant un objet ou un procédé bien défini a pour équivalent plusieurs termes synonymes dans la LA. 


\section{Cas no 4}

Un terme de la LD correspond dans la LA à un terme aux contours différents (ex.: sanitaire/sanitary; pédagogique ).

Cas no 5

Un terme polysémique correspondant à plusieurs dénotés appelle autant de termes différents dans la LA, compte tenu du domaine d'application (ex.: divergence).

Cas no 6

Un même terme a des équivalents différents dans la LA parce qu'il a d'autres antonymes (ex.: eau douce).

\section{Cas no 7}

Un terme de la LD désignant une réalité nouvelle n'a pas encore d'équivalent dans la LA. On entre alors dans le processus de création néologique.

La pratique montre qu'il est souhaitable de ne pas présenter les mots dans leur isolement théorique puisque les termes n'occupent pas une position identique dans la mosaïque des champs lexicaux. L'étudiant s'efforcera donc de délimiter l'extension du terme en en recherchant la définition ou en précisant son environnement contextuel. Ce sont aussi les verbes et adjectifs entourant le substantif technique qui constituent autant de chausse-trapes pour le traducteur. Il est apparu avec évidence qu'il existe dans toute langue de spécialité des «tandems obligés», des combinaisons spécifiques de termes pour décrire certains phénomènes.

\section{La présentation du corpus et des index}

Pour la présentation, le terminologue en herbe se tiendra à la recommandation ISO/R 1149 «Présentation des vocabulaires systématiques multilingues", qui a pour objet de donner une description détaillée des formes de présentation à donner aux vocabulaires systématiques multilingues.

Le mémoire devra toujours comporter:

a) un corpus systématique

b) des index alphabétiques.

L'ordonnance des rubriques du corpus est déterminée d'après la contiguïté des notions. On peut de surcroît introduire un ordre logique dans les rubriques qui reflète les rapports entre les notions incorporées dans le glossaire.

D'après la norme citée, une rubrique doit comprendre:

a) le mot-souche et l'explication de la notion. Une définition des mots clefs est souhaitable, celle-ci ne doit pas nécessairement être établie dans toutes les langues du vocabulaire. Le mot-souche ne doit en aucun cas être une traduction d'une définition d'une langue de départ;

b) un symbole de classification, qui désigne la place d'une notion dans l'ensemble dont elle fait partie. Par exemple: le sous-domaine;

c) une indication sur la portée géographique des termes: $\mathrm{B}, \mathrm{CH}, \mathrm{US}, \mathrm{GB}$; 
d) un numéro de série qui désigne la place d'une rubrique. Il sert à repérer une rubrique dans l'index alphabétique, de même que les renvois, qui sont des termes repris à l'index mais qui n'apparaissent pas sous forme de mots clefs.

Le glossaire peut prendre la forme d'un vocabulaire à rubriques verticales. Chaque rubrique se présente alors sous forme de case, ce qui présente plusieurs avantages: la rubrique peut être vue dans sa totalité d'un seul coup d'œil, le nombre de langues peut être augmenté, les illustrations peuvent être plus facilement incorporées dans la rubrique, enfin les cases peuvent être découpées en fiches. Dans les vocabulaires à rubriques horizontales, les sections monolingues se suivent horizontalement, chacune des langues étant alors placée dans une colonne particulière.

Chaque rubrique sera accompagnée de la référence permettant de retrouver le contexte spécifique, s'il s'agit d'un glossaire «mot pour mot ", et de la source ( nature du document, année de publication ).

Chaque recueil de termes systématiques est assorti d'index alphabétiques contenant tous les termes contenus dans le corpus. Chaque terme y figure nanti du numéro de série renvoyant à la rubrique. Tout terme composé de plusieurs éléments est introduit dans l'index en plusieurs endroits. La répétition dans l'index d'un même terme polysémique est indispensable chaque fois qu'il a pour équivalent plusieurs monosèmes dans une des langues considérées.

\section{Perspectives d'avenir}

Il reste à envisager la mise en valeur des riches matériaux qui s'entassent dans les archives. Jusqu'ici, ils sont venus gonfler les «armoires à mémoires» et les plus nobles intentions d'échange n'ont guère abouti.

On devrait toutefois penser à rassembler par grands thèmes les mémoires élaborés d'abord dans deux langues et à les regrouper en vocabulaires multilingues.

Si plusieurs établissements d'enseignement supérieur font élaborer des mémoires répondant aux mêmes critères, il serait utile de créer un vaste réseau d'échange de ces vocabulaires de spécialité. Toute école associée à ce réseau s'engagerait à confier le résultat de ses travaux à un office international, sorte de Banque des mémoires de terminologie, qui transmettrait aux usagers les éléments nouveaux contenus dans les travaux et qui sont rendus de plus en plus nécessaires par le développement des sciences et du savoir.

Le résultat des recherches serait transmis soit sous forme de table des matières soit sous forme de fiches précisant le sujet étudié, le nombre de termes, etc.

Le mémoire, à la fois monographie et lexique, conçu dans un esprit pragmatique, permettrait peut-être, à sa manière, d'éviter le regrettable hiatus existant, en Europe, entre l'enseignement supérieur et la pratique et de rapprocher l'étudiant de son employeur éventuel.

ROGER GOFFIN 